\title{
Exploring the Structure-Activity Relationship on Platinum Nanoparticles
}

\author{
Laura Braunwarth ${ }^{1}$ (D) Christoph Jung ${ }^{1,2,3}$ (1) $\cdot$ Timo Jacob $^{1,2,3}$ (])
}

Published online: 21 July 2020

(c) The Author(s) 2020

\begin{abstract}
The design of active and stable Pt-based nanoscale electrocatalysts for the oxygen reduction reaction (ORR) plays the central role in ameliorating the efficiency of proton exchange membrane fuel-cells towards future energy applications. On that front, theoretical studies have contributed significantly to this research area by gaining deeper insights and understanding of the ongoing processes. In this work, we present an approach capable of characterizing differently-shaped platinum nanoparticles undergoing thermally- and adsorbate-induced restructuring of the surface. Further, by performing ReaxFF-Grand Canonical Molecular Dynamics simulations we explored the water formation on these roughened ("realistic") nanoparticles in a $\mathrm{H}_{2} / \mathrm{O}_{2}$ environment. Taking into consideration the coverage of oxygen-containing intermediates and occurring surface roughening the nanoparticles' activities were explored. Hereby, we succeeded in locally resolving the water formation on the nanoparticles' surfaces, allowing an allocation of the active sites for $\mathrm{H}_{2} \mathrm{O}$ production. We observed that exposed, low-coordinated sites as well as pit-shaped sites originating from roughening of vertices and edges are most active towards $\mathrm{H}_{2} \mathrm{O}$ formation.
\end{abstract}

Keywords Reactive forcefield $\cdot$ Platinum $\cdot$ Nanoparticles $\cdot$ Oxygen reduction reaction $\cdot$ Structure-activity relationship

\section{Introduction}

Advances in the performance of proton exchange membrane fuel cells (PEMFCs) for economically viable future applications are aimed for in various areas of research, both theoretically and experimentally. As the cathodic oxygen reduction reaction (ORR) on platinum-based electrodes is still considered as one of the bottleneck reactions due to its sluggish kinetics, considerable efforts have been undertaken to its understanding [1]. It has become accepted knowledge that this kinetic hindrance is largely due to oxygenated reaction

Electronic supplementary material The online version of this article (https://doi.org/10.1007/s11244-020-01324-w) contains supplementary material, which is available to authorized users.

Timo Jacob

timo.jacob@uni-ulm.de

1 Institute of Electrochemistry, Ulm University, Albert-Einstein-Allee 47, 89081 Ulm, Germany

2 Helmholtz-Institute Ulm (HIU) Electrochemical Energy Storage, Helmholtz-Straße 16, 89081 Ulm, Germany

3 Karlsruhe Institute of Technology (KIT), P.O. Box 3640, 76021 Karlsruhe, Germany intermediates expressing too strong binding to the platinum electrode, their removal thereby posing the rate and potential limiting step [2,3]. Following the Sabatier principle, the ORR activity is strongly linked to the adsorption energies of its reaction intermediates. Merging these ideas, socalled volcano plots have been established as guideposts for the catalyst design, correlating surface adsorption energies with predictions of the catalytic activity of materials. Based on these correlations, it has been proposed that an optimal catalyst should express a weaker binding towards hydroxide molecules $\left(\mathrm{OH}^{*}\right)$ by $\sim 0.1 \mathrm{eV}$ compared to $\mathrm{Pt}(111)$ [4-8].

Further screening methods, also based on density functional theory (DFT) methods, have been established over the last years, allowing to connect measurable quantities with predictions and trends in the catalytic activity and behavior. One pioneering approach among those methods is surely the $d$-band model by Hammer and Nørskov, aiming at predicting the reactivity of late transition metals based on the $d$-band center of the surface atoms [9].

Also, by applying the concept of generalized coordination numbers $(\mathrm{gCN})$ information about the local structure of catalysts can be obtained and correlated to trends in e.g. adsorption energies [8, 10-12]. Following intuition, the binding energy of oxygenated species should decrease 
with increasing coordination of the active site [13-15]. The gCNs constitute a first-order extension of the usual coordination numbers by including the second nearest neighbors into consideration. Thus, "structure sensitivity" should be captured more thoroughly; in particular, the comparison between nanoparticles and extended surfaces can be improved by covering "finite-size effects". Now, coupling Sabatier's volcano plots with gCNs results in coordination-activity plots, assigning functional groups of $\mathrm{Pt}$ with higher $\mathrm{gCN}$ than the model $\mathrm{Pt}(111)$ surface higher activities $[8,12,16]$. Platinum nanoparticles can enhance the performance of the ORR while their high surface area to volume ratios also reduce catalyst loading. However, the challenge remains to find optimum sizes, shapes and morphologies for an enhanced catalytic process [17-20].

Apart from the previously mentioned criteria for optimized ORR catalysts, long-term stability under the reaction's harsh conditions is of utter importance. Yet, the above-mentioned simple screening models cannot account for thermally- or adsorbate-induced reconstructions of platinum surfaces or nanoparticles and as a consequence thereof lack predictions about possible changes in the catalytic behavior. Alternatively to DFT methods, reactive force field-based approaches such as ReaxFF are predestined to model such structural changes and reactions of extended systems containing several thousand atoms [21-24]. ReaxFF features the description of electronicstructure-dependent chemical properties of atoms, though without an explicit description of individual electrons, and is highly transferable up to complex reaction chemistry at interfaces. An extension to this method is our recently developed Grand Canonical Molecular Dynamics (GCMD) approach that promises to merge reactive molecular dynamics (MD) simulations with constant composition of the system's components, enabling the simulation of catalytic processes near equilibrium [25].

In this work, selected nanoparticles in a hydrogen $\left(\mathrm{H}_{2}\right)$ and oxygen $\left(\mathrm{O}_{2}\right)$ containing environment have been investigated regarding the catalytic formation of water $\left(\mathrm{H}_{2} \mathrm{O}\right)$. In addition, the site-specific preference of the nanoparticles' surfaces-both pristine and after catalysis-towards electrophilic and electrophobic species has been calculated. Our GCMD method allows capturing oxygen-containing intermediate's surface coverages, structural rearrangements as well as roughening of the nanoparticles, both thermally activated and adsorption induced. Applying the $\mathrm{gCN}$ as geometrical descriptor for platinum surface atoms also produced observable trends in nanoparticle roughening. During the occurring surface rearrangement, the activity of water formation is classified according to the $\mathrm{gCN}$ of the platinum surface functional groups, thereby allowing an identification and allocation of active sites.

\section{Computational Details}

\subsection{Simulation Methods}

\subsubsection{ReaxFF Reactive Force Field}

ReaxFF is a versatile reactive force field approach being successfully applied to a wide range of chemical, physical and material science related research topics [21, 23]. It relies on a bond-order-dependent formulation of the potential energy in conjunction with a time-dependent, polarizable charge description. The bond order is updated at every calculated iteration step depending on the local atomic environment, i.e. the respective bond lengths. As such, bond formation as well as bond breaking/dissociation can be captured and therefore entire reactions or chemical processes can be described as well. The ReaxFF potential involves both bonding terms (e.g. bond, angle and torsion contributions) and non-bonding interaction terms (e.g. van der Waals and Coulomb contributions). Atomic charges are derived from the electronegativity equalization method and serve for the description of electrostatic interactions [26]. Our self-developed $\mathrm{Pt} / \mathrm{O} / \mathrm{H}$ force field (details can be found in Refs. [27, 28]) has been used for all simulations. As it has not only been trained and intensely tested against bulk platinum phases, oxygen adsorption and initial stages of platinum oxide formation, but also for energies and reaction barriers along various water formation mechanisms, this forcefield is aptly suited for the here investigated systems. Finally, coverages have been evaluated by analysing the bonding table during the simulation runs.

\subsubsection{Grand Canonical Molecular Dynamics}

Advantageous to simulations of time-dependent properties (such as processes on surfaces) is extending the canonical $N V T$ ensemble ( $N=$ number of particles, $V=$ system volume, $T=$ temperature) by a grand canonical treatment. In our GCMD approach the composition as well as the quantities of the different components in the system are maintained by regularly adjusting the amount of $\mathrm{H}_{2}$ and $\mathrm{O}_{2}$ molecules (i.e. reactants) as well as $\mathrm{H}_{2} \mathrm{O}$ and $\mathrm{H}_{2} \mathrm{O}_{2}$ molecules (i.e. products). In this way, a nearly constant level of partial pressures of $\mathrm{H}_{2}$ and $\mathrm{O}_{2}$ is conserved by adding molecules if necessary and removing product molecules (e.g. $\mathrm{H}_{2} \mathrm{O}$ and $\mathrm{H}_{2} \mathrm{O}_{2}$ ) from the system. As chemical potentials are dependent on temperature and the respective activity, our GCMD approach aims at keeping the chemical potentials constant by firstly controlling the temperature, and secondly by adjusting the partial pressures or activities. Further information and details concerning this method can be found in Ref. [25]. 
Table 1 Description of the applied temperature regimes

\begin{tabular}{llllll}
\hline Temperature setup & $\mathrm{T} 1(\mathrm{~K})$ & $\mathrm{T} 2(\mathrm{~K})$ & $\mathrm{T} 3(\mathrm{~K})$ & $\mathrm{T} 4(\mathrm{~K})$ & $\mathrm{T} 5(\mathrm{~K})$ \\
\hline Pt nanoparticle & 300 & 300 & 300 & 500 & 600 \\
$\mathrm{H}_{2} / \mathrm{O}_{2}$ environment & 1000 & 700 & 600 & 500 & 600 \\
\hline
\end{tabular}

As even ReaxFF simulations can only cover nanoseconds, applying a higher temperature (e.g. attributing a higher kinetic energy to the particles) is a convenient way to overcome higher activation barriers and to effectively sample reaction events. Thus, different temperature setups have been chosen for the present GCMD simulations, as summarized in Table 1: Firstly, the platinum structures have been appointed $300 \mathrm{~K}$, whereas higher temperatures have been assigned to the $\mathrm{H}_{2}$ and $\mathrm{O}_{2}$ molecules of the surrounding atmosphere (T1-T3). These temperature setups have proven to accelerate the establishment of intermediate coverages on the nanoparticle structures, the observation of product formation and the induced structural rearrangement of the nanoparticles. Also, to compensate the high temperature of the gas phase molecules and hence avoiding an excessive preference of desorption processes, the platinum nanoparticles were kept at $300 \mathrm{~K}$, thus providing a corresponding temperature basin. Secondly, temperature setups allocating the same temperatures to the nanoparticle and the $\mathrm{H}_{2}$ and $\mathrm{O}_{2}$ environment (T4-T5) have been applied for an overall comparison of the observed processes and reactions.

Prior to the GCMD simulations presented in the following, the nanoparticle systems in contact with a stochiometric 2:1$\mathrm{H}_{2} / \mathrm{O}_{2}$ atmosphere have been equilibrated ( $N V T$ ensemble) at $300 \mathrm{~K}$ for $125 \mathrm{ps}$. For the environment a relatively high pressure of 50 bar was used, which is justified by the characteristics and limitations of the theoretical approach: Only by increasing the pressure well above experimental conditions, sufficient particle densities could be achieved (36-120 $\mathrm{H}_{2}$ and $\mathrm{O}_{2}$ molecules depending on the system). It should be mentioned that under these simulation conditions, the $\mathrm{H}_{2} / \mathrm{O}_{2}$ environment is still gaseous. After the initial equilibration, the subsequent GCMD simulations ran for $3 \mathrm{~ns}$ (a total of 12 million iterations). All simulations were performed such that after each $10,000 \mathrm{MD}$ iterations, the system was analysed regarding the occurrence of reactant (i.e. $\mathrm{H}_{2}, \mathrm{O}_{2}$ ) and product molecules (i.e. $\mathrm{H}_{2} \mathrm{O}, \mathrm{H}_{2} \mathrm{O}_{2}$ ). Based on this, $\mathrm{H}_{2}$ and $\mathrm{O}_{2}$ molecules were added to the system to maintain their respective partial pressure and present product molecules have been removed from the system, thus keeping the chemical potentials constant. During the reactive MD simulations presented in the following, a timestep of $0.25 \mathrm{fs}$ was employed, utilizing a velocity-Verlet-algorithm. The simulation temperature has been controlled applying a Berendsen thermostat with a temperature-damping constant of $100 \mathrm{fs}$ [29]. All simulations were performed employing ReaxFF implementation within the Amsterdam Density Functional (ADF) package [30, 31]. For visualizations of the nanoparticle structures the program VMD has been utilized [32].

\subsection{Nanoparticle System Setup}

All nanoparticles have been cut from crystalline $f c c$-Pt using the optimized lattice constant of $3.95 \AA$. The cube consists of $\{100\}$ facets, the octahedron possesses $\{111\}$ and the dodecahedron $\{110\}$ facets, whereas the cuboctahedron is made up of both $\{111\}$ and $\{100\}$ and the sphere consists of mixed facets. A diameter around 3-4 $\mathrm{nm}$ has been chosen as a compromise between the observed peak in mass activity at $\sim 3 \mathrm{~nm}$ and to ensure for sufficient stability, as Pt dissolution is more pronounced on small nanoparticles below $4 \mathrm{~nm} \mathrm{[33,}$ 34]. Therefore, the diameter range between 3 and $4 \mathrm{~nm}$ should be catalytically most interesting [35]. The respective diameters of the investigated nanoparticles as well as the respective system sizes and compositions can be found in Table 2 . The used ReaxFF force field's transferability from extended surfaces to nanoparticle structures has been confirmed recently [36]. There, the stability of octahedral, cubic, dodecahedral, cuboctahedral and spherical nanoparticles with sizes ranging between 1 and $10 \mathrm{~nm}$ has been tested, resulting in the stability sequence: cuboctahedron $>$ octahedron $>$ sphere $>$ dodecahedron $>$ cube. In addition, the formation energy of Pt clusters has been compared to corresponding DFT calculations [37], showing good agreement for the particle's size range studied here.

\section{Results and Discussion}

In the following section, we first characterize and discuss the investigated nanoparticles both in their pristine and roughened shapes, enabling the allocation of active sites for water formation in the subsequently performed GCMD simulations. Here, exemplarily only images regarding the cube and
Table 2 The investigated nanoparticle systems are summarized regarding their diameter, number of atoms as well as the system sizes and composition of the $\mathrm{H}_{2} / \mathrm{O}_{2}$ environment

\begin{tabular}{llllll}
\hline Particle shape & Cube & Octahedron & Dodecahedron & Cuboctahedron & Sphere \\
\hline Diameter $(\mathrm{nm})$ & 3.04 & 4.10 & 4.28 & 3.21 & 3.00 \\
Number of Pt-atoms & 665 & 891 & 1163 & 711 & 767 \\
Extension of simulation box $(\mathrm{nm})$ & $4 \times 4 \times 4$ & $6 \times 6 \times 6$ & $6 \times 6 \times 6$ & $4.5 \times 4.5 \times 4.5$ & $4.5 \times 4.5 \times 4.5$ \\
Number of molecules $\left(\mathrm{H}_{2} / \mathrm{O}_{2}\right)$ & $24 / 12$ & $80 / 40$ & $80 / 40$ & $32 / 16$ & $32 / 16$ \\
\hline
\end{tabular}


octahedron are shown, while the respective figures of the other nanoparticle systems can be found in the Supporting Information (SI, references are included in the text).

\subsection{Descriptors for Geometry and Interaction}

\subsubsection{Generalized Coordination Numbers ( $\mathrm{gCN}$ )}

For the characterization of the different nanoparticle shapes and their specific sites the generalized coordination number $(\mathrm{gCN})$ turned out to be a robust and meaningful geometric descriptor. For all systems $\mathrm{gCN}_{i}$, being the generalized coordination number of a surface top site $i$, is calculated as the sum of all first-nearest neighbours' coordination numbers $c n(j)$ divided by the bulk coordination $c n_{\max }$ (e.g. 12 for face centred cubic lattices), see Eq. (1). The respective coordination number, $c n(j)$, of each atom $j$ is calculated as the sum of neighbouring platinum atoms expressing a bond order $\geq 0.3$ to atom $j$.

$\mathrm{gCN}_{i}=\sum_{j=1}^{n_{i}} \frac{c n(j)}{c n_{\max }}$

Characterizing the nanoparticles with this $\mathrm{gCN}$ yields different values for adsorption sites, depending on their vicinity to vertices, edges and facets. For instance, the $\mathrm{gCN}$ of a top site on a $\{111\}$ surface is calculated to be $\mathrm{gCN}_{i}=(6 \cdot 9+3 \cdot 12) / 12=7.5$, summing up six neighbours in the first (each surrounding by 9 atoms) and three neighbours in the second layer (each surrounded by 12 atoms). Hereby, double counting of nearest neighbours is avoided and the $\mathrm{gCN}$ are between 0 and 12 for $f c c$ crystals $[10,13]$. With the $\mathrm{gCN}$ being able to distinguish between adsorption sites on surfaces, it allows estimating the preference of adsorbing species towards certain sites: e.g., hollow sites on the $\{111\}$ can be separated in $h c p(\mathrm{gCN}=7.5)$ and $f c c$ $(\mathrm{gCN}=6.95)$. Thus, the adsorption of electronegative species such as $\mathrm{O}^{*}$ on $f c c$ sites can be identified as adsorption at the three-fold hollow side with lower $\mathrm{gCN}$ value. As for the classical coordination numbers, a $\mathrm{gCN}$ value smaller than the bulk crystal value (e.g. 12 for $f c c$ crystals) is typical for surfaces and describes atoms with a tendency towards the formation of new bonds, allowing to compensate for a lack in coordination. There exists a proportionality between the undercoordination and the inclination to form new bonds, as bond-order conservation theory suggests [10, 38-41]. It needs to be noted, however, that though some trends can be predicted the full description of adsorption behaviour still calls for more sensitive calculations or descriptors [10].

\subsubsection{Solvent Accessible Surface Area (SASA)}

To link the geometric arrangement of the nanoparticles to their estimated adsorption properties, we utilized a fast and efficient screening method. Therefore, we calculated the nanoparticle's solvent-accessible surface area (SASA) and performed a screening regarding the interaction towards atomic oxygen and hydrogen. Here, the interaction signifies (and is a qualitative measure for) the preference of the respective surface site towards an electrophilic (e.g. chargedonating, tested with the oxygen atom) or electrophobic (e.g. charge-accepting, tested with the hydrogen atom) species. Firstly, the SASA is obtained by distributing a set of analysis points along the van der Waals surface of the nanoparticle. Afterwards, at each point the interaction of a probe atom to the particle was evaluated by ReaxFF single point calculations, whereby the corresponding probe atom was placed at a distance of $0.5 \AA$ from the nanoparticle's van der Waals-surfaces. ${ }^{1}$

\subsubsection{Connecting Both Descriptors (gCN and SASA)}

In the following, these two descriptors for geometry and interaction were applied to the pristine, highly-symmetric nanoparticles.

Starting with the cube, the gCN of its surface as well as its preference towards electrophilic and electrophobic intermediates is depicted in Fig. 1. The lowest $\mathrm{gCN}$ values can be found on the cube's vertices (3.17) and on the edges (3.67). According to bond-order preservation theory [40], these sites are expected to express the highest preference towards charge-donating species (e.g. the oxygen probe atom). This is confirmed in Fig. 1b, showing the interaction with the oxygen probe atom.

Subsequently, the inverse relation between $\mathrm{gCN}$ and preference towards electrophilic particles can be observed in Fig. 1 - that is decreasing $\mathrm{gCN}$ should result in increasing bond strengths $[8,10]$. Further, the preference of the Pt particle towards the hydrogen probe atom (being representative for a charge-accepting species) is more pronounced towards the nanoparticle's facets than to its edges and vertices.

For the octahedron, the increasing $\mathrm{gCN}$ values from the edges to the facets can be seen in Fig. S2.1 (see Supporting

\footnotetext{
1 The distance of $0.5 \AA$ between the van der Waals surface of the nanoparticle and the probe atom's atomic center can be translated to a distance of $2.2 \AA$ between the platinum surface atom's center and the probe atom. This corresponds to the adsorption distance of a water molecules on a $\operatorname{Pt}(111)$ surface. It needs to be considered, that the preferences towards electrophilic/electrophobic species determined here are not adsorption energies, as no relaxation of the oxygen/ hydrogen probe atom on the surface is performed. See in the SI, Fig. S1 for an exemplary SASA description.
} 
Fig. 1 a Generalized coordination numbers of the pristine cubic nanoparticle. The gCN of the surface cover a range of 3.17 to 6.67. b Visual representation of the preference of the surface sites towards electrophilic (probing with an oxygen atom) or electrophobic (probing with a hydrogen atom) intermediates

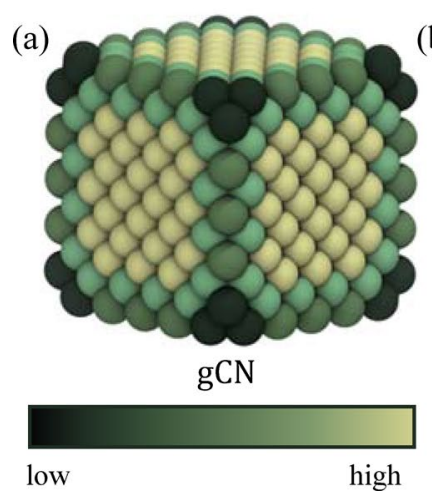

(b)

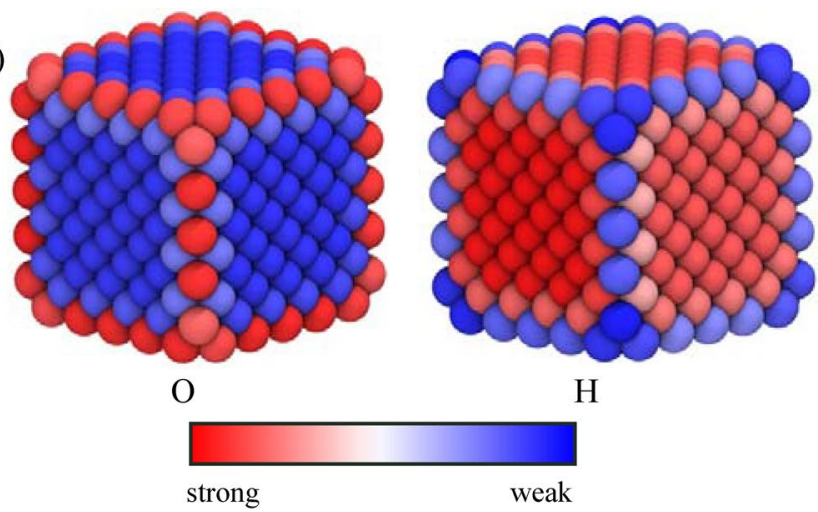

Information (SI)). The facets are even expressing the character of extended $\{111\}$ surfaces, as becomes indicative by a $\mathrm{gCN}$ value of 7.5. As the vertices are displaying $\mathrm{gCN}$ values of 2.33, a strong inclination of bond formation to electrophilic species is expected, which is also confirmed by the vertices' red colouring in Fig. S2.1(b).

On the cuboctahedron, a broader variety of $\mathrm{gCN}$ numbers can be observed (see Fig. S2.3): This can be ascribed to the presence of both $\{111\}$ and $\{100\}$ facets. The characteristic gCN of extended $\{111\}$ surfaces (i.e. 7.5) can be found on the cuboctahedron's $\{111\}$ facets, however, the $\{100\}$ facets are too small (e.g. maximum $\mathrm{gCN}=6.67$ ) to reproduce the behaviour of the extended $\{100\}$ facets that would have $\mathrm{gCN}=8.0$. A distinguishable preference towards electrophilic (resp. electrophobic) species can be observed between the $\{100\}$ and $\{111\}$ facets and the vertices between them (see Fig. S3(b)).

Regarding the dodecahedron, when comparing the $\mathrm{gCN}$ values of the nanoparticle's facets with the $\mathrm{gCN}$ value of an extended $\{110\}$ surface (i.e. 7), Fig. S2.2 shows that the facets display a maximum gCN values of 5.87. Thus, one would expect a higher preference towards charge-donating species than on the extended $\{110\}$ surface. From Fig. S2.2(b) it can also be concluded, that the underlying second layer is not accessible for adsorbates or intermediates, as the preference towards the oxygen and hydrogen probe atom is weak.

The spherical nanoparticle consists of mixed facets and therefore also displays a wide variety of $\mathrm{gCN}$ values, see Fig. S2.4 (a). The atoms that are located at exposed sites are displaying the lowest gCN numbers as well as the highest preference towards electrophilic species, as determined by the oxygen probe atom.

\subsection{Surface Roughening During Operation}

Surface roughening of nanoparticle structures needs to be considered when accounting for the harsh catalytic conditions during the ORR [42]. While the roughening process or degradation may lead to numerous different particle structures, shapes and sizes, whose full analysis is out of the scope of molecular dynamics simulations, it is still possible to study the interplay between surface structure and intermediates. For instance, exposing the pristine, highly-symmetric nanoparticles to the hydrogen and oxygen-containing environment and the different temperature setups during our GCMD simulations, various effects of surface roughening could be observed on the investigated nanoparticle shapes. Again, the gCN as well as the preference of the surface sites towards a charge-donating or -accepting probe atom served as structural descriptors. The GCMD simulations have been performed for $3 \mathrm{~ns}$, during which the distribution of the nanoparticle's gCN numbers have been determined within two timeframes. In the following, $t_{0}$ represents the starting point of the GCMD, $\Delta t_{1}$ covers the interval $0.75-1.5 \mathrm{~ns}$ and $\Delta t_{2}$ covers the second interval between 2.25 and $3.0 \mathrm{~ns}$. The gCN distributions have then been averaged over the respective intervals and yield information about the time-dependent structural shape of the nanoparticles. As can be seen in Fig. 2a, the pristine cube (prior to the GCMD simulations, e.g. at $t_{0}$ ) features distinct gCN values. However, the clear attribution of the gCN to structural features becomes difficult already during the first time interval $\Delta t_{1}$. Overall, one can see that the thermally- and/or adsorbate- induced local surface rearrangements cause a broadened distribution of gCNs.

Hereby, the peak corresponding to the gCN number of $\{100\}$ facets (e.g. 7.0) shrinks considerably, while gCN values laying between the identified numbers of facets, vertices and edges appear. Remarkably, roughening of the cube's vertices also causes the appearance of gCN values $\leq 2$, suggesting a strong preference towards charge-donating species. During the second time interval $\left(\Delta t_{2}\right)$ these low gCN values become even more frequent. Also, gCN values attributed to subsurface atoms become blurred. Along with the changes in $\mathrm{gCN}$ distribution, changes in the preference towards the oxygen and hydrogen probe atom are of interest when aiming at deeper insights into the 


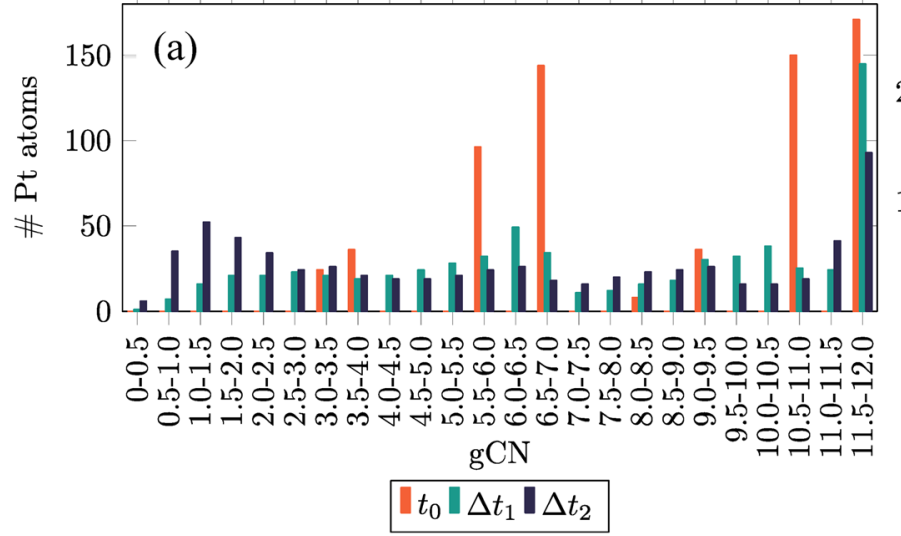

Fig. 2 Time-dependent $\mathrm{gCN}$ distribution at different snapshots along the GCMD simulation. The nanoparticle shapes were analyzed initially at $\mathrm{t}_{0}$, averaged between 0.75 and $1.5 \mathrm{~ns}$ simulation time

catalytic behaviour. As can be seen in Fig. 3, the exposed and strongly roughened vertices of the cube feature these low $\mathrm{gCN}$ values $\leq 2$ as well as a stronger preference towards electrophilic and electrophobic species than the few remaining ordered facets. However, pit-shaped local arrangements (indicated by black arrows in Fig. 3b) also display an increased preference towards charge-donating or -accepting species. These shapes represent a concave site, already postulated to be more active towards the ORR by Calle-Vallejo et al. [15] The influence of the different temperature setups on the observed surface roughening is included in the SI exemplary for the cubic nanoparticle. As to be expected, the degree of the observed phenomena correlates with the applied temperature, however, the previously mentioned features subsist generally.

For the octahedron, the peak corresponding to $\mathrm{gCN}=7.5$ (i.e. the $\{111\}$ facets) is reduced by $\sim 90 \%$ until the end of the 3 ns long GCMD simulation (see Fig. 2b). This is mainly caused by adsorbate-induced surface buckling, where a platinum surface atom is pushed out of the surface plane to prevent oxygen-containing intermediates (e.g. $\mathrm{OH}^{*}$, $\mathrm{OOH}^{*}, \mathrm{O}^{*}$ ) laying too close to one another, thus reducing electrostatic repulsion [27]. The surface buckling observed on the $\{111\}$ facets of the octahedron also creates sites with increased preference towards electrophilic species, see the exposed red atoms in Fig. S3.2(b). Apart from the buckling, the $\{111\}$ facets are mostly maintained. As observed on the cube, roughening up of the edges causes the appearance of lower gCN numbers.

The $\{110\}$ facets of the dodecahedron appear to be less stable than the $\{111\}$ facets during the GCMD simulations: Structural rearrangements affect the anisotropic structure of the first and second layer and are thereby creating a lowsymmetry, rough surface (see Fig. S3.3). Concave sites (as already mentioned on the cubic nanoparticle) can also be (b)

200

100

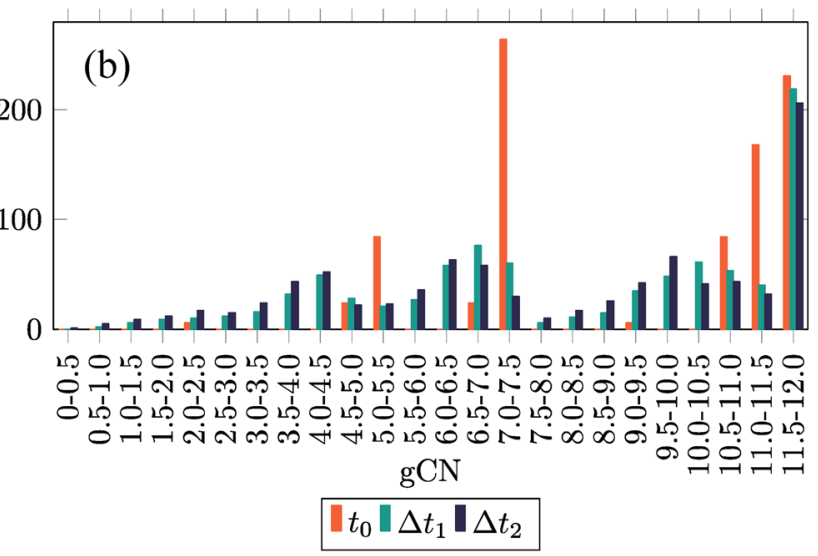

(labelled as $\Delta t_{1}$ ) and finally averaged between 2.25 and $3 \mathrm{~ns}$ simulation time (labelled as $\Delta t_{2}$ ); $\mathbf{a}$ cube and $\mathbf{b}$ octahedron. Temperature setup $\mathrm{T} 1$ is evaluated

observed on the dodecahedron, where the rearrangement of atoms belonging to the $\{110\}$ facets near vertices is inclined to create pits.

The cuboctahedron, featuring both $\{111\}$ and $\{100\}$ facets expresses a similar behavior as observed on the cube and on the octahedron: The roughening of the edges invokes the occurrence of $\mathrm{gCN} \leq 2$, whereas the facets remain mainly intact, apart from local surface buckling (see Fig. S3.4). Those structural changes have resulted in exposed platinum atoms as well as pit-shaped sites near vertices displaying a strong preference towards charge-donating species.

On the spherical nanoparticle an almost even distribution of gCN values between 0.5 and 11.0 can be observed in $\Delta t_{1}$ (see Fig. S3.1). After $3 \mathrm{~ns}$, only a minor increase of $\mathrm{gCN}$ values $<3.5$ can be seen. Due to the sphere's mixed facet character, it features many pit-shaped arrangements of platinum surface atoms (see Fig. S3.5).

In general, all investigated nanoparticles express a broadened distribution of gCNs with tails both towards smaller values and higher values caused by surface roughening during the GCMD simulations. On all nanoparticles, the breaking up of the ordered nanoparticle structures in particular starts at vertices and to a lesser degree at edges. The more distinct the vertices (e.g. cube or octahedron) the more intense the roughening.

\subsection{Locally Resolved Water Formation}

\subsubsection{Coverage of Intermediates}

Another feature that needs to be taken into consideration when studying the catalytic behaviour of structures is the coverage of intermediates and adsorbates during the processes, as the dynamically changing local atomic environment is presumably influencing the catalytic properties. 
(a)

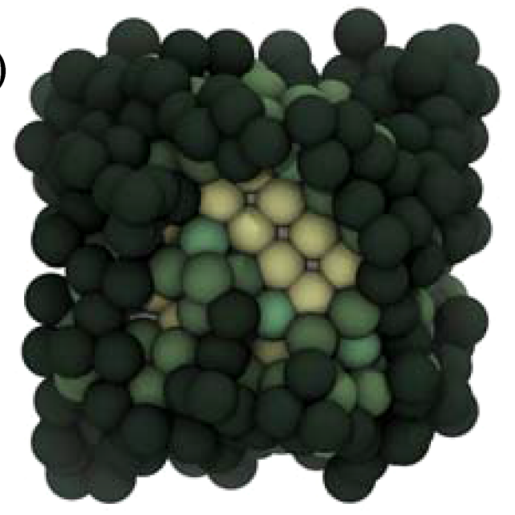

$\mathrm{gCN}$

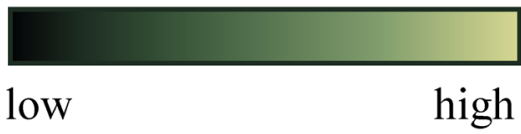

Fig. 3 a Generalized coordination numbers of the roughened cubic nanoparticle. The gCN are colored according to the groups: $0-2,2-4$, 4-6 and 6-8. b Visual representation of the preference of the surface sites towards electrophilic (probing with an oxygen atom) or electro-

However, these cannot be captured by descriptors solely focusing on a single binding energy per species. During our performed GCMD simulations, surface coverages of intermediates (e.g. $\mathrm{O}^{*}, \mathrm{OH}^{*}, \mathrm{OOH}^{*}$ ) indeed fluctuated over time and caused structural rearrangements, as previously discussed (e.g. surface roughening and buckling). In the following, the surface composition of the nanoparticles is briefly discussed. Hereby, to allow for a comparison between the different nanoparticles, the coverage is measured in number of adsorbate molecules per surface area $\left(\mathrm{nm}^{2}\right)$, see Table 3 . The respective van der Waals surfaces of the roughened $\mathrm{Pt}$ nanoparticles have been obtained by the SASA method. ${ }^{2}$

Starting with the cubic nanoparticle, $\mathrm{OH}^{*}$ and $\mathrm{O}^{*}$ show the highest coverages. Though the coverage of $\mathrm{O}^{*}$ is higher than $\mathrm{OH}^{*}$ (see Table 3), these $\mathrm{O}^{*}$ are mainly incorporated into the roughened edges and vertices and should therefore be seen as surface oxide-species rather than adsorbates. $\mathrm{OH}^{*}$ is covering the whole nanoparticle surface, on the exposed sites of strongly roughened vertices one can even observe multiple $\mathrm{OH}^{*}$ per platinum atom. Additionally, $\mathrm{OH}^{*}$ is encircling the pit-shaped arrangements.

On the octahedron, $\mathrm{OH}^{*}$ is covering the nanoparticle in loose chains or ring-like structures. $\mathrm{O}_{2} *$ is mainly adsorbed on the edges while $\mathrm{O}^{*}$ is incorporated in the roughened sites. Also, $\mathrm{OOH}^{*}$ is adsorbed on edges and the roughened vertices.

\footnotetext{
2 The GCMD simulations of the different nanoparticle systems applying temperature setup $\mathrm{T} 1$ are evaluated in the following. All values have been determined at the end of the simulations, e.g. after $3 \mathrm{~ns}$.
}

phobic (probing with a hydrogen atom) intermediates. Only platinum atoms are shown, adsorbates are not depicted. Temperature Setup T1 has been applied

Similar observations can be made on the dodecahedron, with $\mathrm{OH}^{*}$ forming chains on the topmost layers of the $\{110\}$ facets and decorating exposed platinum atoms or island on the surface. No oxygen-containing intermediate is adsorbed on the second layer, as predicted by the SASA descriptor. $\mathrm{O}_{2}{ }^{*}$ adsorption is more pronounced on the edges than on the facets.

Regarding the cuboctahedron, $\mathrm{OH}^{*}$ is covering edges and facets likewise, on some exposed platinum surface atoms multiple $\mathrm{OH}^{*}$ are adsorbed. $\mathrm{O}^{*}$ is incorporated in the roughened vertices and edges. The same trends can be found for the sphere with $\mathrm{O}_{2} *$ and $\mathrm{OH}^{*}$ decorating the exposed sites while $\mathrm{OH}^{*}$ is covering the whole nanoparticle surface. Also, $\mathrm{O} *$ is involved in the roughening process.

On all nanoparticle structures, $\mathrm{OH}^{*}$ is the predominant intermediate during the GCMD simulations: Its coverage is steadily increasing, however, near the end of the simulations its increase slows. These mentioned observations are in their character observable in all applied temperature setups; however, their degree differs.

\subsubsection{Activity of Water Formation}

Also, the activity of the nanoparticle differs clearly, as can be seen in Table 4. To allow for a comparison between the different nanoparticles, the activity is measured as number of produced $\mathrm{H}_{2} \mathrm{O}$ molecules per $\mathrm{ns}$ and $\mathrm{nm}^{2}$. The cubic nanoparticle appears to be the most active one towards water formation, showing the highest value of $\mathrm{H}_{2} \mathrm{O}$ production. The cuboctahedron and sphere perform moderate, expressing 
Table 3 Coverages of intermediates (oxygencontaining species) measured at the end of the GCMD simulations (after $3 \mathrm{~ns}$ ) on the platinum nanoparticles

\begin{tabular}{|c|c|c|c|c|c|}
\hline Particle shape & Cube & Octahedron & Dodecahedron & Cuboctahedron & Sphere \\
\hline$\# \mathrm{OH}^{*}\left(\right.$ per $\left.\mathrm{nm}^{2}\right)$ & 3.04 & 3.07 & 2.52 & 2.71 & 3.49 \\
\hline$\# \mathrm{O}^{*}\left(\right.$ per $\left.\mathrm{nm}^{2}\right)$ & 4.02 & 0.51 & 0.65 & 2.67 & 1.73 \\
\hline$\# \mathrm{OOH}^{*}\left(\right.$ per $\left.\mathrm{nm}^{2}\right)$ & 0.04 & 0.45 & 0.32 & 0.13 & 0.11 \\
\hline$\# \mathrm{O}_{2} *\left(\right.$ per $\left.\mathrm{nm}^{2}\right)$ & 0.96 & 1.90 & 2.45 & 1.41 & 1.63 \\
\hline
\end{tabular}

Temperature setup $\mathrm{T} 1$ is evaluated a production rate $50 \%$ below the cube's one. Lastly, the octahedron and dodecahedron show the lowest number of produced $\mathrm{H}_{2} \mathrm{O}$ molecules, with the octahedron forming only $\sim 25 \%$ of the amount of water molecules compared to the cube and the dodecahedron only $\sim 10 \%$, respectively. Remarkably, the selectivity of all investigated nanoparticles towards $\mathrm{H}_{2} \mathrm{O}$ formation (with $\mathrm{H}_{2} \mathrm{O}_{2}$ as side-product) is higher than on extended surfaces (see for comparison Ref. [25]), with the cube and the sphere showing the highest $\mathrm{H}_{2} \mathrm{O}: \mathrm{H}_{2} \mathrm{O}_{2}$ ratios.

These observations can only carefully be compared to experimental findings. Our simplifications made (e.g. the lack of electrolyte) complicates the rating of the nanoparticle's activity: Sánchez-Sánchez et al. [43] discovered the dependence of the $\{111\}$ and $\{100\}$ facet activity of the anion present in solution. Other computational approaches, like the density functional theory study performed by Mahata et al. [44], investigating the stability, ORR mechanisms and kinetic aspects, attribute the cuboctahedral nanoparticle a higher activity and selectivity compared to the octahedron. This is mirrored in our observed activities.

\subsubsection{Allocation of Water Formation Sites}

After applying the geometric descriptors for the characterization of the roughened nanoparticle structures discussed in the previous section, the nanoparticle's actual catalytic behavior was then investigated during the GCMD simulations. Here, the activity of water formation has been classified according to the $\mathrm{gCN}$ of the platinum atoms, thereby allowing identifications of active sites. The produced $\mathrm{H}_{2} \mathrm{O}$ molecules have been traced during the MD simulation regarding their metallic binding partners, whose respective $\mathrm{gCN}$ values have been calculated. Thereby, the $\mathrm{H}_{2} \mathrm{O}$ production could be allocated to certain $\mathrm{gCNs}$. This evaluation has been performed separately during the time frames $\Delta t_{1}$ $(0.75-1.5 \mathrm{~ns})$ and $\Delta t_{2}(2.25-3 \mathrm{~ns})$ of the GCMD simulations. For all nanoparticles, three GCMD simulations have been performed at each temperature setup T1-T5. Then, the formation of the water molecules has been traced in all simulations. The combined results of all simulations of one nanoparticle (for the respective time frame) were used to generate the respective locally resolved activity diagram. These diagrams show the number of produced $\mathrm{H}_{2} \mathrm{O}$ molecules per $\mathrm{gCN}$ number (scaled by its frequency on the nanoparticle) and allow the allocation and comparison of active sites regarding water formation.

For the cubic nanoparticle, the locally resolved activity diagram is displayed in Fig. 4a. Two distinct trends can be observed: First, water formation is performed rather at the cube's edges and vertices than at facets (as the $\mathrm{gCN}$ would suggest), though those sites have already undergone slight structural rearrangements. Second, with increasing roughness of the surface (evaluating $\Delta t_{2}$ ) the $\mathrm{H}_{2} \mathrm{O}$ formation preferentially occurs at surface sites characterized by even lower $\mathrm{gCN}$ values $(\leq 2)$. Such low $\mathrm{gCN}$ values can be found at the exposed, low-symmetric sites of the cube (as depicted in Fig. 3a).

For the octahedron and cuboctahedron, the sites featuring $\mathrm{gCN}$ values in the range 1.0-2.5 are also the most active with respect to $\mathrm{H}_{2} \mathrm{O}$ formation. On the octahedron (see Fig. 3b), sites with low gCN values are formed due to surface buckling on the $\{111\}$ facets, therefore, these buckled platinum atoms seem to be active for water formation as well. Additionally, for $\mathrm{gCN}$ values around 7 the resolved water formation activity is slightly enhanced with increasing simulation time: These sites can be attributed to pit-shaped formations, emerging due to the roughening processes, or intact $\{111\}$ facets. As the roughening of the nanoparticle increases with time, it is more probable for the pit-shaped formations to express the water forming activity as the $\{111\}$ facets are expected to lose their integrity. On the cuboctahedron (see Fig. S5.1(b)), the water formation activity can be mainly attributed to roughened edges, as those are expressing the lowest $\mathrm{gCN}$ values.

However, regarding the dodecahedron, no clear preferences can be observed: Sites characterized by $\mathrm{gCN}$ values between 0.5 and 7 contribute to the product formation. The same behavior can be observed for the sphere (see 
Table 4 Activity of the investigated nanoparticles regarding the formation of $\mathrm{H}_{2} \mathrm{O}$ and $\mathrm{H}_{2} \mathrm{O}_{2}$

\begin{tabular}{|c|c|c|c|}
\hline Nanoparticle & $\begin{array}{l}\text { Surface } \\
\text { area }\left(\mathrm{nm}^{2}\right)\end{array}$ & $\begin{array}{l}\# \mathrm{H}_{2} \mathrm{O} \\
\left(\text { per ns nm }{ }^{2}\right)\end{array}$ & $\begin{array}{l}\# \mathrm{H}_{2} \mathrm{O}_{2} \\
(\text { per ns nm²) }\end{array}$ \\
\hline Cube & 53.97 & 2.87 & 0.01 \\
\hline Octahedron & 48.91 & 0.66 & 0.17 \\
\hline Dodecahedron & 60.32 & 0.30 & 0.04 \\
\hline Cuboctahedron & 45.39 & 1.18 & 0.03 \\
\hline Sphere & 52.68 & 1.10 & 0.01 \\
\hline
\end{tabular}

The simulation time between 2.25 and $3 \mathrm{~ns}\left(\Delta t_{1}\right)$ has been evaluated and the numbers have been scaled regarding the van der Waals surface area (obtained by the SASA method) of the roughened nanoparticles. Temperature setup T1 is evaluated

Fig. S5.1(c)), though here a tendency towards lower $g C N s$ becomes more pronounced when evaluating the second time frame $\Delta t_{2}$, compared to $\Delta t_{1}$.

Comparing all investigated nanoparticles regarding their resolved local water forming activity, two general observations can be made: Active sites towards $\mathrm{H}_{2} \mathrm{O}$ formation are on the one hand exposed platinum surface atoms, being found on the roughened edges and vertices of the nanoparticles or on buckled surface atoms. These exposed sites feature low $\mathrm{gCN}$ values and are decorated with oxygen-containing intermediates (e.g. $\left.\mathrm{OH}^{*}\right)$. Second, pit-shaped sites being characterized by $\mathrm{gCN}$ values around 7 , mostly located near roughened edges or vertices, also pose active water forming sites. As even the GCMD can only capture a few nanoseconds, the formation of the pit-shaped sites may become more important due to an enhanced surface roughening with time.

These findings are in line with conclusions drawn from experiments: The importance of investigating the effects of heat treatment on platinum nanoparticles has been stressed by Aarons et al. [45], observing surface roughening on $\mathrm{Pt}$ nanoparticles going along with a general lowering of the surface gCN. This is further supported by Chung et al. [46], attributing the nanoparticle surficial rearrangement a beneficial contribution to the ORR activity, the degree of which being affected by the heat treatment conditions.

\section{Conclusion}

Transferring structural properties to catalytic behaviour plays a key role in designing catalytic systems. In this work, we have illustrated how gas-phase platinum nanoparticles are modified in their surface morphology by the catalytic conditions and based on this, how these rough, low-symmetry nanoparticles behave regarding water formation. Hereby, we have taken into consideration the influence of oxygen containing intermediates on the surfaces. We could observe that edges and vertices are prone to surface roughening, while $\{111\}$ facets are experiencing surface buckling. These exposed, low-coordinated sites have been detected as the most active regions towards water formation. Another feature, caused by surface roughening, is the occurrence of pit-shaped sites. While being characterized by $\mathrm{gCN}$ values around 7, these also express an increased preference towards electrophilic and electrophobic species and pose another region active for water formation. Moreover, the effects associated with adsorbate-induced or thermally-activated processes causing a broadening of the $\mathrm{gCN}$ distribution (i.e. transferable to a roughening of the nanoparticle structures), are already occurring at temperatures of 500-600 K. Therefore, it is of utter importance to further elucidate the properties and catalytic behaviour of realistic platinum nanoparticles. Hereby, the theoretical-computational approaches

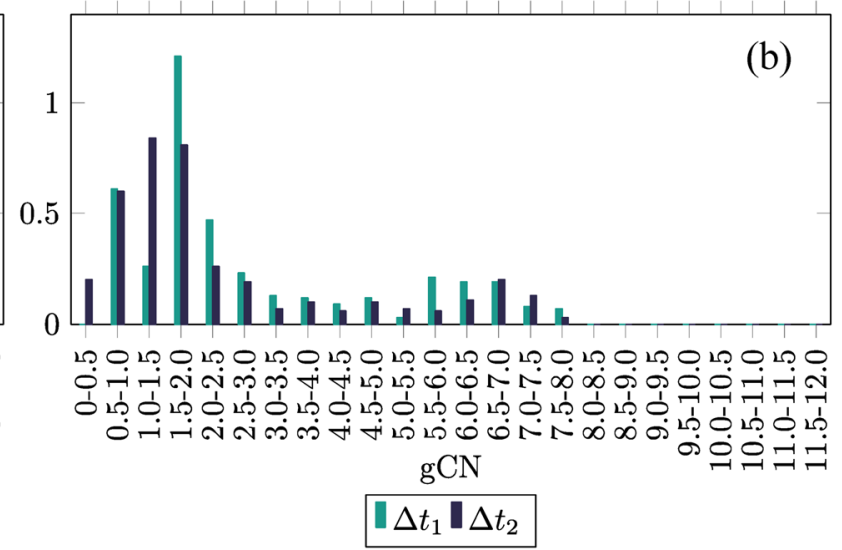

is obtained. $\Delta t_{1}$ covers the simulation time between 0.75 and $1.5 \mathrm{~ns}$, while $\Delta t_{2}$ covers 2.25 to $3 \mathrm{~ns}$ : a cube and $\mathbf{b}$ octahedron. Calculated over T1-T5
Fig. 4 Produced $\mathrm{H}_{2} \mathrm{O}$ molecules have been tracked to their presumable formation site. By dividing the total number of $\mathrm{H}_{2} \mathrm{O}$ per $\mathrm{gCN}$ by the number of $\mathrm{Pt}$ atoms with that $\mathrm{gCN}$ the locally resolved activity 
can be envisioned to further enhance the understanding of those catalysts.

Acknowledgements Open Access funding provided by Projekt DEAL. This work was supported by the German Research Foundation (DFG) under Project ID 390874152 (POLiS Cluster of Excellence) as well as the Sonderforschungsbereich (collaborative research centre) SFB-1316. Further, support from the BMBF (Bundesministerium für Bildung und Forschung) through the project GEP ("Fundamentals of electrochemical interfaces", Grant Agreement: 13XP5023D) is gratefully acknowledged. Finally, the authors acknowledge the computer time supported by the state of Baden-Württemberg through the bwHPC project and the DFG through Grant No. INST40/467-1 FUGG. A special thanks to Björn Kirchhoff for supplying the nanoparticle structures.

\section{Compliance with Ethical Standards}

Conflict of interest The authors declare that they have no conflict of interest.

Open Access This article is licensed under a Creative Commons Attribution 4.0 International License, which permits use, sharing, adaptation, distribution and reproduction in any medium or format, as long as you give appropriate credit to the original author(s) and the source, provide a link to the Creative Commons licence, and indicate if changes were made. The images or other third party material in this article are included in the article's Creative Commons licence, unless indicated otherwise in a credit line to the material. If material is not included in the article's Creative Commons licence and your intended use is not permitted by statutory regulation or exceeds the permitted use, you will need to obtain permission directly from the copyright holder. To view a copy of this licence, visit http://creativecommons.org/licenses/by/4.0/.

\section{References}

1. Stephens IEL, Rossmeisl J, Chorkendorff I (2016) Science 354:1378-1379

2. Nørskov JK, Rossmeisl J, Logadottir A, Lindqvist L, Kitchin JR, Bligaard T, Jónsson H (2004) J Phys Chem B 108:17886-17892

3. Rossmeisl J, Karlberg GS, Jaramillo T, Nørskov JK (2009) Faraday Discuss 140:337-346

4. Greeley J, Stephens IEL, Bandarenka AS, Johansson TP, Hansen HA, Jarmillo TF, Rossmeisl R, Chorkendorff I, Nørskov JK (2009) Nat Chem 1:552-556

5. Stephens IEL, Bondarenko AS, Perez-Alonso FJ, Calle-Vallejo F, Bech L, Johansson TP, Jepsen AK, Frydendal R, Knudsen BP, Rossmeis1 J, Chorkendorff I (2011) J Am Chem Soc 133:5485-5491

6. Stephens EL, Bandarenka AS, Grønbjerg U, Rossmeisl J, Chorkendorff I (2012) Energy Environ Sci 5:6744-6762

7. Escudero-Escribano M, Malacrida P, Hansen MH, Vej-Hansen UG, Velázquez-Palenzuela A, Tripkovic V, Schiøtz J, Rossmeisl J, Stephens IEL, Chorkendorff I (2016) Science 352:73-76

8. Calle-Vallejo F, Tymoczko F, Colic V, Vu QH, Pohl MD, Morgenstern K, Loffreda D, Sautet P, Schuhmann W, Bandarenka AS (2015) Science 350:185

9. Hammer B, Nørskov JK (2000) Adv Catal 45:71-129
10. Calle-Vallejo F, Martínez JI, García-Lastra JM, Sautet P, Loffreda D (2014) Angew Chemie Int Ed 53:8316-8319

11. Calle-Vallejo F, Loffreda D, Koper MTM, Sautet P (2015) Nat Chem 7:403-410

12. Calle-Vallejo F, Pohl MD, Reinisch D, Loffreda D, Sautet P, Bandarenka AS (2017) Chem Sci 8:2283-2289

13. Rossi K, Asara GG, Baletto F (2019) Phys Chem Chem Phys 21:4888

14. Asara GG, Paz-Borbón LO, Baletto F (2016) ACS Catal 6:4388-4393

15. Kortlever R, Shen J, Schouten KJP, Calle-Vallejo F, Koper MTM (2015) J Phys Chem Lett 6:4073-4082

16. Rück M, Bandarenka A, Calle-Vallejo F, Gagliardi A (2019) Nanoscale Adv 1:2901

17. Schlögl R (2015) Angew Chem Int Ed 54:3465-3520

18. Lee I, Delbecq F, Morales R, Albiter MA, Zaera F (2009) Nat Mater 8:132-138

19. Strasser P (2015) Science 349:379-380

20. Cui C, Gan L, Heggen M, Rudi S, Strasser P (2013) Nat Mater 12:765-771

21. Senftle TP, Hong S, Islam MM, Kylasa SB, Zheng Y, Shin YK, Junkermeier C, Engel-Herbert R, Janik MJ, Aktulga HM, Verstraelen T, Grama A, van Duin ACT (2016) Npj Comput Mater 2:15011

22. Han Y, Jiang D, Zhang J, Li W, Gan Z, Gu J (2016) Front Chem Sci Eng 10:16-38

23. van Duin ACT, Dasgupta S, Lorant F, Goddard WA (2001) J Phys Chem A 105(41):9396-9409

24. Chenoweth K, van Duin ACT, Goddard WA (2008) J Phys Chem A 112:1040-1053

25. Jung CK, Braunwarth L, Jacob T (2019) J Chem Theory Comput 15(11):5810-5816

26. Mortier WJ, Ghosh SK, Shankar S (1986) J Am Chem Soc 108:4315-4320

27. Fantauzzi D, Bandlow J, Sabo L, Mueller JE, van Duin ACT, Jacob T (2014) Phys Chem Chem Phys 16:23118-23133

28. Fantauzzi D, Mueller JE, Sabo L, van Duin ACT, Jacob T (2015) ChemPhysChem 16:2797-2802

29. Berendsen HJC, Postma JPM, van Gunsteren WF, DiNola A, Haak JR (1984) J Chem Phys 81:3684

30. te Velde G, Bickelhaupt FM, Baerends EJ, Fonseca-Guerra C, van Gisbergen SJA, Snijders JG, Ziegler T (2001) J Comput Chem 22:931-967

31. Baerends EJ et al ADF2017, SCM, Theoretical chemistry. Vrije Universiteit, Amsterdam, The Netherlands, https://www.scm.com

32. Humphrey W, Dalke A, Schulten K (1996) J Mol Graph 14:33-38

33. Tritsaris GA, Greeley J, Rossmeisl J et al (2011) Catal Lett 141:909

34. Gasteiger HA, Kocha SS, Sompalli B, Wagner FT (2005) Appl Catal B 56:9-35

35. Meier JC et al (2014) Beilstein J Nanotechnol 5:44-67

36. Kirchhoff B, Braunwarth L, Jónsson H, Fantauzzi D, Jacob T (2020) Small 16:1905159

37. Lambie SG, Weal GR, Blackmore CE, Palmer RE, Garden AL (2019) Nanoscale Adv 1:2416-2425

38. Groß A (2008) J Comput Theor Nanosci 5:894-922

39. Peterson I, Grabow L, Brennan T, Shong B, Ooi C, Wu D, Li C, Kushwaha A, Medford A, Mbuga F, Li L, Nørskov J (2012) Top Catal 55:1276-1282

40. Shustorovich E, Sellers H (1998) Surf Sci Rep 31:1-119 
41. Kleis J, Greeley J, Romero NA, Morozov VA, Falsig H, Larsen AH, Lu J, Mortensen JJ, Dułak M, Thygesen KS, Nørskov JK, Jacobsen KW (2011) Catal Lett 141:1067-1071

42. Markovic NM et al (2014) Energy Environ Sci 7:4061

43. Sanchéz-Sanchéz IM, Solla-Gullón J, Vidal-Iglesias FJ, Aldaz A, Montiel V, Herrero E (2010) J Am Chem Soc 132(16):5622-5624

44. Mahata A, Rawat KS, Choudhuri I, Pathak B (2016) Catal Sci Technol 6:7913
45. Aarons J, Jones L, Varambhia A, MacArthur KE, Ozkaya D, Sarwar M, Skylaris C-K, Nellist PD (2017) Nano Lett 17:4003-4012

46. Chung IY, Chung Y-H, Jung N, Choi K-H, Sung Y-E (2013) Phys Chem Chem Phys 15:13658-13663

Publisher's Note Springer Nature remains neutral with regard to jurisdictional claims in published maps and institutional affiliations. 